\title{
A universal, multifunctional, high-practicability superhydrophobic paint for waterproofing grass houses
}

Hai Zhu' ${ }^{1}$ Yu Huang ${ }^{1,2}$, Shouwei Zhang ${ }^{1}$, Shiwei Jin $^{3}$, Xiaoding Lou ${ }^{1}$ and Fan Xia $\mathbb{C l}^{1}$

\begin{abstract}
Roof leakage is a common phenomenon on rainy days and makes residents uncomfortable. Superhydrophobic materials are promising candidates to protect grass houses from rainwater. However, mechanical weakness, chemical corrosion, and UV light sensitivity are the three main challenges restricting these nonwetting materials from wider application in real life. Herein, we developed an inorganic-organic superhydrophobic paint (IOS-PA) for preparing a waterproof grass house. IOS-PA not only showed mechanical robustness and chemical anticorrosion but also displayed self-healing properties, anti-icing properties, and high and low temperature $\left(150^{\circ} \mathrm{C}\right.$ and $\left.-196^{\circ} \mathrm{C}\right)$ resistance. Photocatalysis was also achieved with IOS-PA, as demonstrated by organic matter (Nile red, methyl blue, and methyl orange) degradation. Moreover, extremely long-term UV resistance, i.e., resistance to UV irradiation ( $365 \mathrm{~nm}, 5.0 \pm$ $0.6 \mathrm{~mW} / \mathrm{cm}^{2}$ ) for $100 \mathrm{~h}$ and ambient sunlight for $8640 \mathrm{~h}$ (1 year), caused the conflicting properties of superhydrophobicity and photocatalysis to coexist in IOS-PA, further accomplishing self-cleaning for the removal of both dirt particles and organic contamination. Specifically, a grass house coated with IOS-PA exhibited favorable waterproof properties, indicating the potential to ensure comfortable living conditions for people living in undeveloped areas, even on rainy days. With a variety of excellent characteristics, IOS-PA, we believe, is advantageous for scalable production and practical application in reality.
\end{abstract}

\begin{tabular}{ll}
\multicolumn{2}{l}{ Abbreviations } \\
IOS-PA & Inorganic-organic superhydrophobic paint \\
PTFE & Polytetrafluoroethylene \\
NPs & Nanoparticles \\
PDMS & Polydimethylsiloxane \\
ER & Epoxyresin \\
PFOS & 1H,1H,2H,2H-perfluorooctyltriethoxysilan \\
SEM & Scanning Electron Microscopes \\
TEM & Transmission electron microscopy \\
XPS & X-ray photoelectron spectroscopy \\
XRD & X-Ray Diffraction \\
WCA & Water contact angle \\
RA & Rolling-off angle
\end{tabular}

Correspondence: Fan Xia (xiafan@cug.edu.cn)

${ }^{1}$ Engineering Research Center of Nano-Geomaterials of Ministry of Education, Faculty of Materials Science and Chemistry, China University of Geosciences, Wuhan 430074, P. R. China

${ }^{2}$ Zhejiang Institute, China University of Geosciences, Hangzhou 311305, P. R. China

Full list of author information is available at the end of the article
MB
MO
Methyl blue
GDP Methyl orange
Gross Domestic Product 
houses for real-world applications outdoors, three main challenges must be addressed: (I) mechanical weakness $^{10,11}$, (II) chemical corrosion ${ }^{12}$, and (III) UV sensitivity $^{13}$. Micro- and/or nanostructures provide surface roughness, but they wear easily under mechanical forces. To achieve mechanical superhydrophobicity, the flexible "coating + adhesive" method has been proven to be of significance and has indicated the potential for wide application $^{14,15}$. An all-organic water-repellent coating containing resin polymer and polytetrafluoroethylene nanoparticles (PTFE NPs) was developed that exhibited mechanochemical robustness resulting from the mechanical and chemical properties of the nanocomposite constituents ${ }^{16}$. UV light is a harmful factor for superhydrophobic materials ${ }^{17,18}$. By mixing metal oxide photocatalysts with hydrophobic polymers, such as PTPE $\mathrm{NPs}^{19}$, polydimethylsiloxane (PDMS) ${ }^{20,21}$ and silicone nanofilaments ${ }^{22}$, (super)hydrophobic surfaces with longterm anti-ultraviolet properties have been prepared.

To face much more complex environments in practice, in addition to addressing the above three challenges, various functions, such as a large coating area ${ }^{14-16}$, selfhealing properties ${ }^{23,24}$, anti-icing properties ${ }^{25-27}$, highand low-temperature resistance ${ }^{11,28-30}$, photocatalysis $^{31,32}$ and self-cleaning properties ${ }^{14,33-35}$, are strongly advocated for these nonwetting materials. However, although the aforementioned features have been achieved on diverse substrates in recent years, the combination of all of these features on a universal superhydrophobic surface or coating has yet to be reported.

In this study, by mixing dual-sized $\mathrm{TiO}_{2} \mathrm{NPs}$ with epoxy resin (ER) followed by grafting $1 \mathrm{H}, 1 \mathrm{H}, 2 \mathrm{H}, 2 \mathrm{H}$-perfluorooctyltriethoxysilan (PFOS), we prepared an inorganicorganic superhydrophobic paint (IOS-PA) (Scheme 1). IOSPA featured mechanical robustness and anticorrosion arising from the mechanochemically stable ER. Self-healing properties, anti-icing properties, high-temperature $\left(150^{\circ} \mathrm{C}\right)$ and liquid nitrogen $\left(-196^{\circ} \mathrm{C}\right)$ resistance, and self-cleaning properties were also observed for IOS-PA. Furthermore, under UV illumination, IOS-PA showed efficient photodegradation of organic dyes, while super water repellency was still maintained even under exposure to UV light (365 $\mathrm{nm}, 5.0 \pm 0.6 \mathrm{~mW} / \mathrm{cm}^{2}$ ) for $100 \mathrm{~h}$ and ambient sunlight for $8640 \mathrm{~h}$ (1 year). With its UV-resistant water repellency, mechanical robustness, chemical anticorrosion properties, self-healing properties, resistance to low- and hightemperature damage, anti-icing properties, photocatalysis, and self-cleaning properties, our multifunctional IOS-PA indicates the potential for necessary, meaningful, and practical applications in waterproofing houses in undeveloped areas.

\section{Experimental procedures}

Materials

The epoxy resin and curing agent were obtained from Beijing Oriental Yuhong Waterproof Technology Co. Ltd.

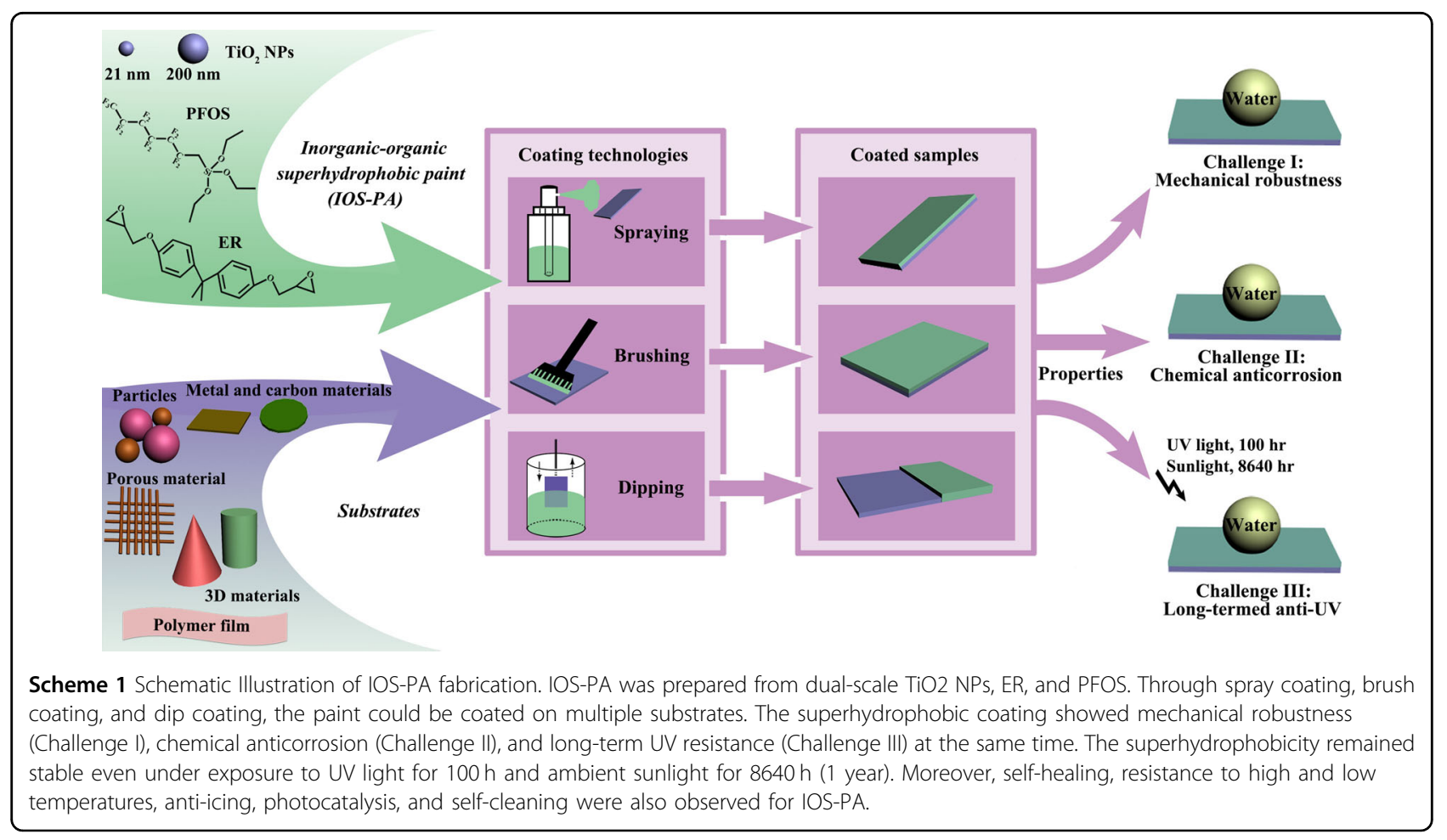


P25 NPs with diameters of approximately $21 \mathrm{~nm}$ and $\mathrm{TiO}_{2}$ (anatase) NPs with diameters of approximately $200 \mathrm{~nm}$ $(100 \mathrm{~nm}$ to $300 \mathrm{~nm})$ were purchased from Evonki Degussa and Aladdin, respectively. PFOS was obtained from Innochem. Nile red powder was obtained from Aladdin. Methyl blue and methyl orange were purchased from Chinese Medicine. Grass houses made of straw and maize leaves were purchased from Taobao Shop.

\section{Fabrication of IOS-PA}

Six grams of epoxy resin was dissolved and stirred in $30 \mathrm{ml}$ of acetone for $15 \mathrm{~min}$, and $6 \mathrm{~g}$ of $\mathrm{TiO}_{2}(21 \mathrm{~nm})$ and $6 \mathrm{~g}$ of $\mathrm{TiO}_{2}(200 \mathrm{~nm})$ dissolved in $150 \mathrm{ml}$ of acetone containing NPs were mixed and stirred for $15 \mathrm{~min}$. Then, the above two solutions were mixed, stirred, and sonicated for two 15-min cycles. Then, $1.2 \mathrm{~g}$ of PFOS was added to the mixed solution, stirred, and sonicated for eight 15 -min cycles. Finally, $0.6 \mathrm{~g}$ of curing agent and $0.2 \mathrm{~g}$ of PFOS were added to the above solution, stirred, and sonicated for two 10-min cycles. Thus, IOS-PA was successfully synthesized. Twenty-four substrates were coated with the super-water-repellent paint by spray coating.

\section{Durability of IOS-PA \\ Mechanical destruction}

Sandpaper abrasion and sand impact were used to test the mechanical robustness of our coated samples. The coated samples were placed face down on sandpaper in NO. A total of 320 or $200 \mathrm{~g}$ of weight was placed on the surface and moved for a distance of $10 \mathrm{~cm}$ with a velocity of approximately $7.5 \mathrm{~cm} / \mathrm{s}$. In every cycle, the water contact angle (WCA) on the surface was measured with $10 \mu \mathrm{L}$ of water drops at five different positions. For the sand impact test, $15 \mathrm{~g}$ of sand particles held in a funnel were dropped onto the prepared surface 50 times from a height of $15 \mathrm{~cm}$, and then, the WCA was tested.

\section{Chemical corrosion}

In the chemical corrosion experiment, the WCAs and rolling-off angles (RAs) of our coating samples were tested after immersion in solutions of $\mathrm{pH}=1$ for $4 \mathrm{~h}, \mathrm{pH}=7$ for $8 \mathrm{~h}$, and $\mathrm{pH}=14$ for $8 \mathrm{~h}$.

\section{Self-healing}

Treatment with $\mathrm{O}_{2}$ plasma $(1 \mathrm{~min})$ and boiling water ( $5 \mathrm{~min}$ ) was conducted to assess the self-healing properties of our coating. Superhydrophobicity was regained after heating in a $150^{\circ} \mathrm{C}$ oven for $5 \mathrm{~min}$.

\section{Anti-icing}

A water drop was placed on a fresh surface or coated surface at $-10^{\circ} \mathrm{C}$. Then, the process of liquid water becoming ice was observed, and the freezing time was recorded.

\section{Low- and high-temperature treatment}

The superhydrophobic coating surface was placed in an oven at $150^{\circ} \mathrm{C}$ for $100 \mathrm{~h}$, and the surface WCA and RA were measured every $10 \mathrm{~h}$. Two hundred milliliters of liquid nitrogen, serving as an extremely cold substance, was poured on the coated samples, and then the WCA and RA were measured after drying at room temperature for 10 repeats.

\section{Photocatalytic activity and UV resistance}

To show the photocatalytic performance, three organic dyes, Nile red, methyl blue, and methyl orange, were used with stirring at $500 \mathrm{rpm}$. Nile red was dissolved in ethanol at a concentration of $10 \mu \mathrm{g} / \mathrm{L} . \mathrm{MB} /$ water $(40 \mu \mathrm{g} / \mathrm{L})$ and $\mathrm{MO} /$ water $(40 \mu \mathrm{g} / \mathrm{L})$ were prepared before mixing with ethanol (volume, water:ethanol $=2: 1$ ). First, $0.06 \mathrm{~g}$ of our coating particles was added to these three solutions and then placed under UV illumination $\left(5.0 \pm 0.6 \mathrm{~mW} / \mathrm{cm}^{2}\right)$ before dark treatment for $30 \mathrm{~min}$. The degradation of the dyes was judged by the disappearance of color and ultraviolet spectrophotometry. Moreover, organic dyes were dropped onto the coated samples, in which were then exposed to UV light. The colors were decomposed with almost no observable color. Our coated samples were placed under UV light $\left(5.0 \pm 0.6 \mathrm{~mW} / \mathrm{cm}^{2}\right)$ and ambient sunlight at the Xuehai Building, China University of Geosciences (Wuhan), from Nov. 1st, 2018, to Nov. 1st, 2019, to test the UV resistance.

\section{Instrumentation}

Surface nanostructures were observed by a HITACHI SU8010 field-emission scanning electron microscope (FESEM, $20 \mathrm{kV}$ ). The complex structures between the ER and $\mathrm{TiO}_{2} \mathrm{NPs}$ were demonstrated by transmission electron microscopy (Tecnai G2 20 S-TWIN). To analyze the chemical composition and surface element content of the samples, X-ray photoelectron spectroscopy (XPS, ESCA$\mathrm{LAB} \mathrm{Xi}+$, Thermo Fisher Scientific) with an $\mathrm{Al} \mathrm{K \alpha}$ line as the excitation source was adopted. X-ray diffraction (XRD, Bruker D8 Advance) was performed using a Dmax$3 \beta$ diffractometer with nickel-filtered $\mathrm{Cu} K \alpha$ radiation to distinguish the crystal forms of titanium dioxide (rutile and anatase). WCA and RA measurements were performed by a DSA100 contact angle analyzer with $10 \mu \mathrm{L}$ water droplets at five different positions to obtain the average contact angle values. Water droplets with a volume of $10 \mu \mathrm{L}$ and a height of $15 \mathrm{~mm}$ were dripped at a velocity of $0.25 \mathrm{~m} / \mathrm{s}$ to show the bouncing phenomena on the coated samples. UV intensity was tested by an ultraviolet radiometer (HANDY). The concentration of Nile red in the presence of our coating and UV light was measured with an ultraviolet spectrophotometer (SHIMADZU). By using a paramagnetic resonance spectrometer (ESR/EPR, Bruker A300), superoxide and hydroxyl 
free radicals from the ER, PFOS- $\mathrm{TiO}_{2} \mathrm{NPs}$, and PFOS-ER$\mathrm{TiO}_{2}$ NPs were studied in an ethanol solution for UV illumination for $10 \mathrm{~min}$.

\section{Results and discussion}

To synthesize the superhydrophobic paint, epoxy resin/ acetone $(6 \mathrm{~g} / 30 \mathrm{ml})$ and dual-scale $\mathrm{TiO}_{2} \mathrm{NPs} /$ acetone $(6 \mathrm{~g}: 6 \mathrm{~g} / 100 \mathrm{ml})$ were mixed, stirred, and sonicated. Subsequently, $1.2 \mathrm{~g}$ of PFOS was added to the above solution to decrease the surface free energies of ER and the $\mathrm{TiO}_{2}$ NPs. Finally, $0.6 \mathrm{~g}$ of curing agent and $0.2 \mathrm{~g}$ of PFOS were added to this composite solution to achieve IOS-PA. In this work, spray coating was used to prepare superhydrophobic samples in all subsequent experiments unless otherwise specified. Various substrates were coated by IOS-PA, including "OD" particles $\left(\mathrm{MnO}_{2}, \mathrm{CuO}, \mathrm{SiO}_{2}\right.$, and sand) (Supplementary Fig. S1), 2D carbon-based substrates (fabric, wood, A4, and filter paper) (Supplementary Fig. S2), 2D silicon-based substrates (ceramic wafers and glass slides) (Supplementary Fig. S3), 2D metal substrates ( $\mathrm{Zn}$ and $\mathrm{Al}$ plates) (Supplementary Fig. S4), 2D polymer films (polyvinyl chloride (PVC), polyethylene (PE) and polystyrene (PS) films and culture dishes) (Supplementary Fig. S5), 3D materials (tetrahedral, cylindrical, and circular cone shapes) (Supplementary Fig. S6), porous substrates (stainless steel mesh and sponges) (Supplementary Fig. S7) and natural leaves (maple, apricot, and loquat leaves) (Supplementary Fig. S8). The coating of a large area by IOS-PA was accomplished on a PS plate with dimensions of $0.5 \times 1 \mathrm{~m}^{2}$ (Supplementary Fig. S9 and Movie S1). It has been previously proven that surface superhydrophobicity can be achieved with an optimal mass ratio of $\mathrm{TiO}_{2}(21 \mathrm{~nm}): \mathrm{TiO}_{2}(200 \mathrm{~nm})=1: 1$. [10] Herein, a mass ratio of $\mathrm{ER}^{\mathrm{TiO}} \mathrm{Ti}_{2}(21 \mathrm{~nm}): \mathrm{TiO}_{2}$ $(200 \mathrm{~nm})=1: 1: 1$ was adopted since the coexistence of superhydrophobicity (Supplementary Fig. S10-S12), photocatalysis (Supplementary Fig. S13), and UV resistance (Supplementary Fig. S14) could be perfectly maintained (Supplementary Table S1, Fig. S15). Two kinds of $\mathrm{TiO}_{2}$ NPs were observed by scanning electron microscopy (SEM) (Fig. 1a) and were confirmed to be coated with ER according to transmission electron microscopy (TEM) images (Fig. 1b-c). As indicated by X-ray photoelectron spectroscopy (XPS) analysis (Fig. 1d), PFOS was grafted onto ER (Supplementary Fig. S16-S17) and the $\mathrm{TiO}_{2} \mathrm{NPs}$ (Fig. 1e and Supplementary Fig. S16) to provide a low surface energy. Anatase and rutile $\mathrm{TiO}_{2} \mathrm{NPs}$ in our coating were also demonstrated by X-ray diffraction (XRD) (Supplementary Fig. S18). Thus, the textured nanostructures and low surface energy of IOS-PA endowed the substrates with superhydrophobic properties, displaying high WCAs $>150^{\circ}$ and low RAs $<10^{\circ}$ (Supplementary Fig. S19 and Movie S2). To demonstrate water droplet bouncing phenomena and dynamic contact angles, five kinds of coated substrates (glass slide, fabric, wood, PS, and ceramic wafer) were utilized. Due to the air films formed over rough structures ${ }^{36-38}$, water droplets (release height $=15 \mathrm{~mm}, v=0.25 \mathrm{~m} / \mathrm{s}$ ) completely left the surfaces without wetting (Fig. 1f). Moreover, the coated surface repelled the impact of water drops released from a height of approximately $80 \mathrm{~cm}$ with different dripping velocities $(v=0.25 \mathrm{~m} / \mathrm{s}, 1 \mathrm{~m} / \mathrm{s}, 2 \mathrm{~m} / \mathrm{s}$, and $3 \mathrm{~m} / \mathrm{s}$ ) (Supplementary Movie S3). Although raindrops with a velocity of approximately $9 \mathrm{~m} / \mathrm{s}$ hit our coated surface, strong water-repellent properties were still observed (Supplementary Figs. 20-21, Movie S4).

Durable superhydrophobic materials are required for practical applications. Mechanical robustness, chemical anticorrosion properties, self-healing properties, antiicing properties, and high- and low-temperature resistance functioned well on IOS-PA. To display the mechanical stability of IOS-PA, sandpaper abrasion and sand impact approaches were applied. A coated glass slide was placed face down on sandpaper (No. 320) (Supplementary Fig. S22), a weight of $200 \mathrm{~g}$ was placed on the reverse side, and then a force was used to push the coated surface and weight for a distance of $10 \mathrm{~cm}$ with a velocity of approximately $7.5 \mathrm{~cm} / \mathrm{s}$ (Supplementary Fig. S23-S24). After 50 repeats, the WCA of the sample was still larger than $150^{\circ}$ (Fig. 2a), showing abrasion-resistant superhydrophobicity. Regarding sand impact destruction, $15 \mathrm{~g}$ of sand with diameters ranging from $0.5 \mathrm{~mm}$ to $2 \mathrm{~mm}$ fell from a height of $15 \mathrm{~cm}$ to hit the surface (Supplementary Fig. S25-S26), and super water repellency of the samples was sustained with WCAs $>150^{\circ}$ after 50 cycles (Fig. 2b). Upon mechanical damage, the original surface morphology changed but still had textured structures, thus offering sufficient roughness to ensure super-nonwetting behavior, mainly due to the favorable adhesive and mechanically robust properties of $\mathrm{ER}^{14,16,39-41}$. In addition to the glass slide-based superhydrophobic surface, the coated fabric, PS, wood, and ceramic wafer substrates exhibited stable superhydrophobic behavior after sandpaper abrasion and sand impact.

After storage in acidic $(\mathrm{pH}=1)$ solution for $4 \mathrm{~h}$ and saline $(\mathrm{pH}=7)$ and alkaline $(\mathrm{pH}=14)$ solutions for $8 \mathrm{~h}$, high WCAs and low RAs remained on coated samples (Fig. 2c-e and Supplementary Fig. S27). Thus, IOS-PA exhibited stable water repellency against chemical corrosion, originating from the inherent chemical inertness of $\mathrm{ER}^{16,39-41}$. Moreover, low-surface-energy compounds on nonwetting materials are often sensitive to outside stimuli, and thus, self-healing water-repellent materials are required $^{23,24}$. IOS-PA manifested superior self-healing ability against oxygen plasma (Fig. $2 \mathrm{f}$ and Supplementary Fig. S28) and boiling water vapor treatments (Supplementary Fig. S29). Self-healing proceeded for 10 cycles of these treatments with no obvious morphology variations, 


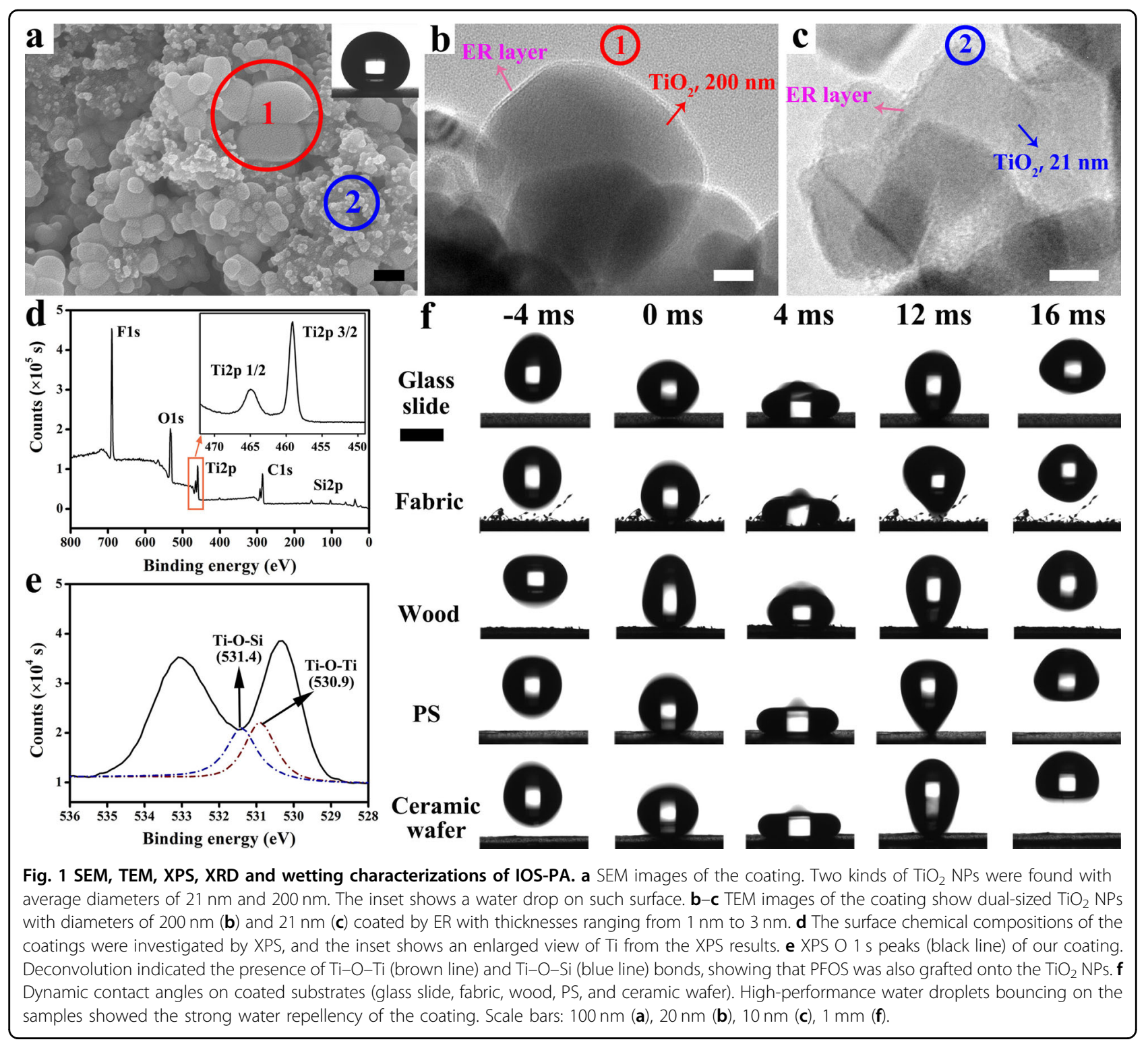

and super water repellency could be regained by treatment at $150{ }^{\circ} \mathrm{C}$ for $5 \mathrm{~min}$ due to the thermostable properties of the material (Fig. $2 \mathrm{~g}$ and Supplementary Fig. S30). IOS-PA also featured favorable anti-icing properties, attributed to the thermal barriers on the textured nanostructures and the small contact region between the surface and liquid ${ }^{26}$. Taking the glass slide as an example (Supplementary Fig. S31a), it took approximately $30 \mathrm{~s}$ for a water droplet (volume: $10 \mu \mathrm{L}$ ) to freeze into ice on the bare surface $\left(-10^{\circ} \mathrm{C}\right)$ (Supplementary Movie S5). However, for the superhydrophobic sample under the same conditions, the freezing time was $120 \mathrm{~s}$ (Supplementary Movie S6). Additionally, ice formation delays were observed on the other four coated samples, i.e., fabric, PS, wood, and the ceramic wafer (Supplementary Fig. S31b). An ice cube easily rolled away from the surface at room temperature (Supplementary Fig. S31c). Notably, even when the coated filter paper (Supplementary Movie S7) and sponge (Supplementary Movie S8) were exposed to extremely cold liquid nitrogen (LN), they still manifested strong water repellency (Supplementary Fig. S32). The WCA and RA on the surface remained almost unchanged after liquid nitrogen treatment (Fig. 2h), indicating outstanding resistance of the composite coating to low temperature.

Under UV illumination, excited superoxide and hydroxyl free radicals from $\mathrm{TiO}_{2}$ NPs can degrade organic substances (Fig. 3a-b and Supplementary Fig. S33) ${ }^{18,19,31,32}$. To demonstrate the photocatalytic properties of IOS-PA, Nile red solutions without and with our coating (PFOS$\mathrm{ER}-\mathrm{TiO}_{2} \mathrm{NPs}$ ) were placed under UV irradiation $(365 \mathrm{~nm}$, $5.0 \pm 0.6 \mathrm{~mW} / \mathrm{cm}^{2}$ ). As illustrated in Supplementary Fig. 


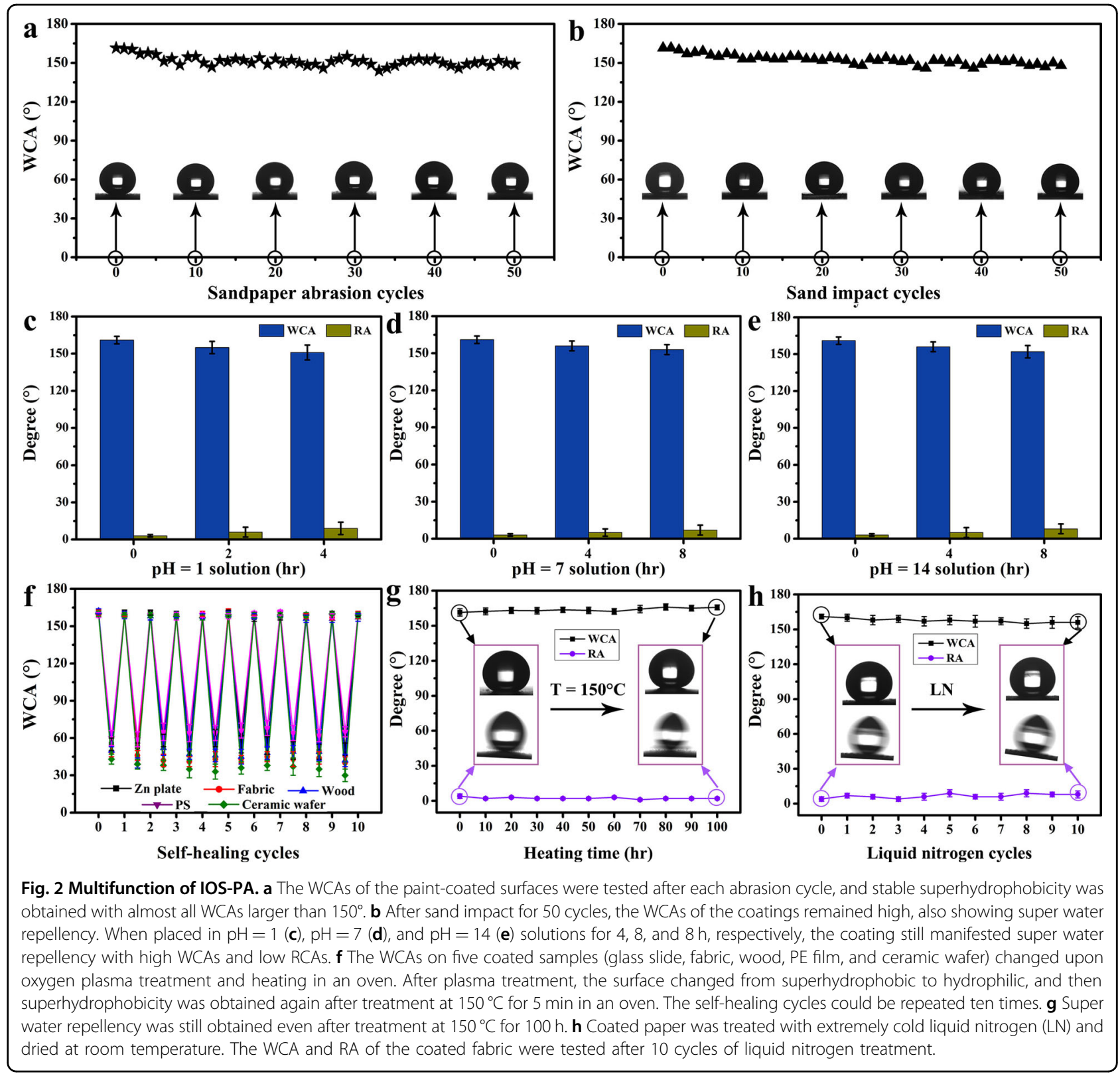

S34, the bare Nile red solution did not show obvious color variation, and a high chemical concentration was still observed under UV irradiation. For PFOS-ER-TiO ${ }_{2} \mathrm{NPs}$, the red dye was almost completely decomposed in $4 \mathrm{~h}$, as further proven by the UV-Vis spectra (Fig. 3c). After 10 repeats, efficient degradation of Nile red was still stably achieved (Fig. 3d). Moreover, eight other kinds of coated samples were used to show photocatalytic activity, i.e., fabric, filter, A4 paper, a culture dish, PE, PVC and PS films and natural loquat leaf; all Nile red-contaminated samples remained clean after UV irradiation (Supplementary Fig. S35). Even in ambient sunlight, organic dye decomposition was observed on the coated substrates (Supplementary Fig. S36). In our composite coating, this high-performance photocatalytic effect was obtained solely because of the existence of dual-sized $\mathrm{TiO}_{2} \mathrm{NPs}$ (Supplementary Fig. S34 and S37) since ER showed no photocatalytic ability (Supplementary Fig. S38). In addition to Nile red decomposition, methyl blue (MB) and methyl orange (MO) were degraded by IOS-PA (Supplementary Fig. S39-S40). The coated surfaces showed robust superhydrophobic properties when exposed to mechanical destruction, chemical corrosion, and liquid nitrogen. Here, when subjected to the same damage, favorable photocatalytic activity was still observed for our coating (Supplementary Fig. S41).

In the outside environment, long-term sunlight illumination often damages superhydrophobicity because 

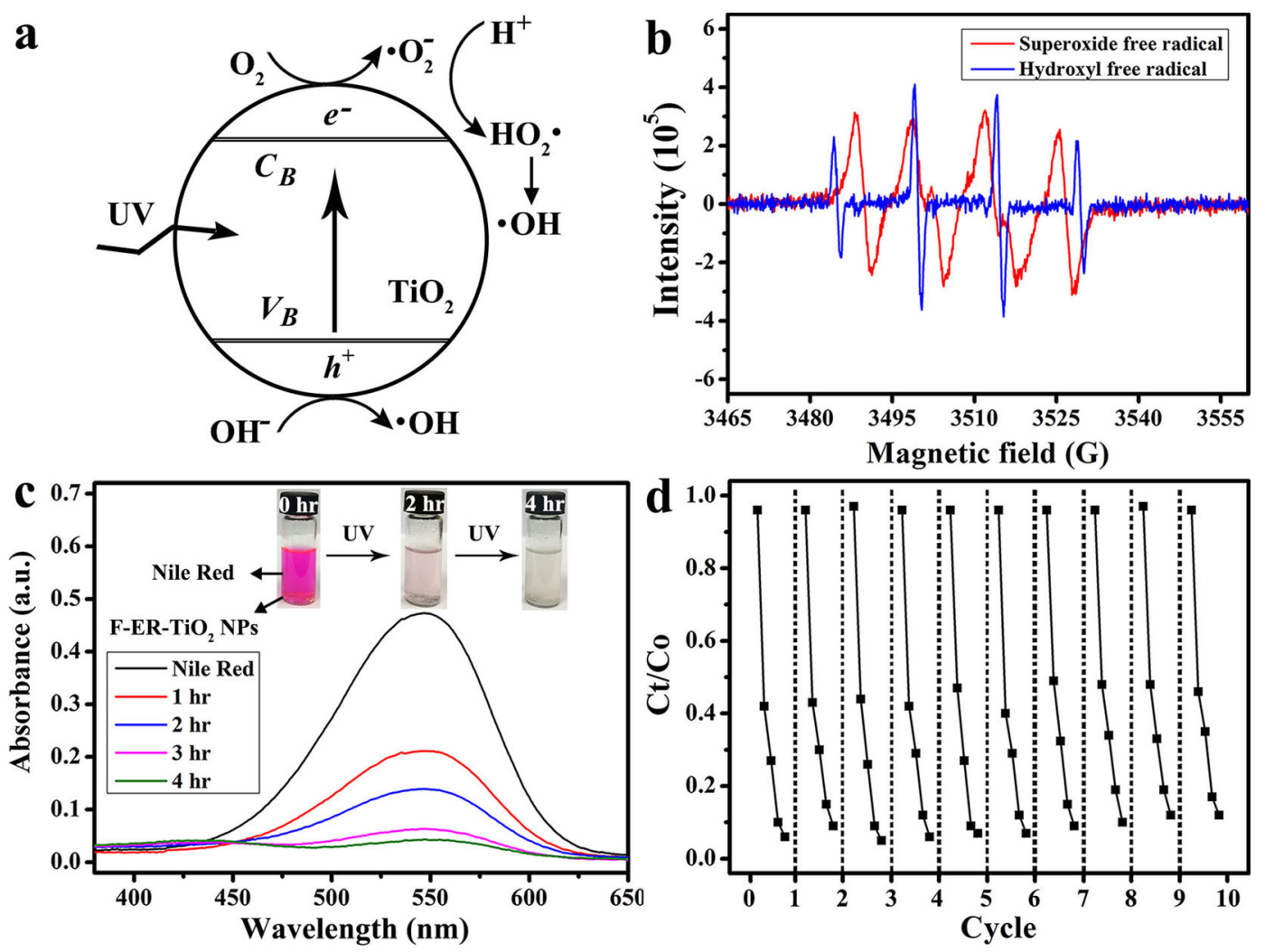

Fig. 3 Photocatalysis of IOS-PA. a Photocatalytic mechanism of $\mathrm{TiO}_{2}$. b Paramagnetic resonance spectrometry (ESR/EPR) spectra of the composite coating; many superoxide free radicals and hydroxyl free radicals were found. c UV-Vis spectra of Nile red solution showing decomposition by F-ER$\mathrm{TiO}_{2} \mathrm{NPs}$ every $1 \mathrm{~h}$. The insets are optical photos of the color variations. d F-ER-TiO 2 NPs showed favorable degradation performance of Nile red for 10 cycles over $4 \mathrm{~h}$. Ct indicates the organic content upon UV illumination for 0, 1, 2, 3, and $4 \mathrm{~h}$ in each cycle, and Co is the original content of Nile red.

interfacial compositions and structures are sensitive to UV light; thus, UV-resistant superhydrophobicity is required for outdoor applications. Specifically, for photocatalysts, (super)hydrophobicity could not be achieved for a long time since the low-surface-energy compounds that were used to reduce surface energy were easily degraded by excited free radicals under UV light ${ }^{17,18}$. Therefore, water-repellent photocatalysts often require dark storage or protection from light. For our coating, superhydrophobicity with a high WCA $>150^{\circ}$ and low RA $<20^{\circ}$ (Supplementary Fig. S42) was achieved under UV irradiation $\left(365 \mathrm{~nm}, 5.0 \pm 0.6 \mathrm{~mW} / \mathrm{cm}^{2}\right)$ for a period of $100 \mathrm{~h}$ (Fig. 4a) with unaffected surface structures (Fig. 4b) and a high content of F (Fig. 4c). When the coated samples were exposed to ambient sunlight for as long as $8640 \mathrm{~h}$ (1 year), they also possessed strong water repellency (Fig. 4d and Supplementary Fig. S42), resulting from their stable surface roughness (Fig. 4e) and chemical composition (Fig. 4f). In IOS-PA, fluorosilane was grafted onto ER and the $\mathrm{TiO}_{2}$ NPs to obtain a low surface energy (Supplementary Fig. S43), which combined with the sufficient roughness derived from the dual-sized $\mathrm{TiO}_{2} \mathrm{NPs}$ to achieve super-nonwetting properties. However, for $\mathrm{TiO}_{2}$
NPs directly modified with PFOS (PFOS- $\mathrm{TiO}_{2} \mathrm{NPs}$ ), superhydrophobicity changed into superhydrophilicity under both UV light and sunlight illumination (Supplementary Fig. S44a-S44c and S45, Table S2) because PFOS was decomposed by the $\mathrm{TiO}_{2}$ NPs. However, fluorinated ER (PFOS-ER) with hydrophobicity (Supplementary Fig. S46) exhibited almost unchanged wetting behavior (Supplementary Fig. S44d) even in the presence of UV light and $\mathrm{TiO}_{2} \mathrm{NPs}$, where almost no obvious variations were found in the surface structure (Supplementary Fig. S44e and S46) or chemical composition (Supplementary Fig. S44f). Hence, PFOS on ER was protected from superoxide and hydrogen free radicals excited by $\mathrm{TiO}_{2} \mathrm{NPs}$, which contributed to the durable low surface energy of IOS-PA and thus long-term super water repellency.

Lotus-inspired superhydrophobic surfaces ${ }^{4,10,14,33-35}$ and (super)hydrophilic metal oxide photocatalysts ${ }^{19-22}$ are two kinds of self-cleaning materials. The first kind of surface can remove dirt (sand, dust, particles, etc.) with the aid of strong water repellency. The latter has been applied to decompose organic contamination by excited free radicals. However, in the presence of both dirt and organic contamination, the above self-cleaning surfaces 


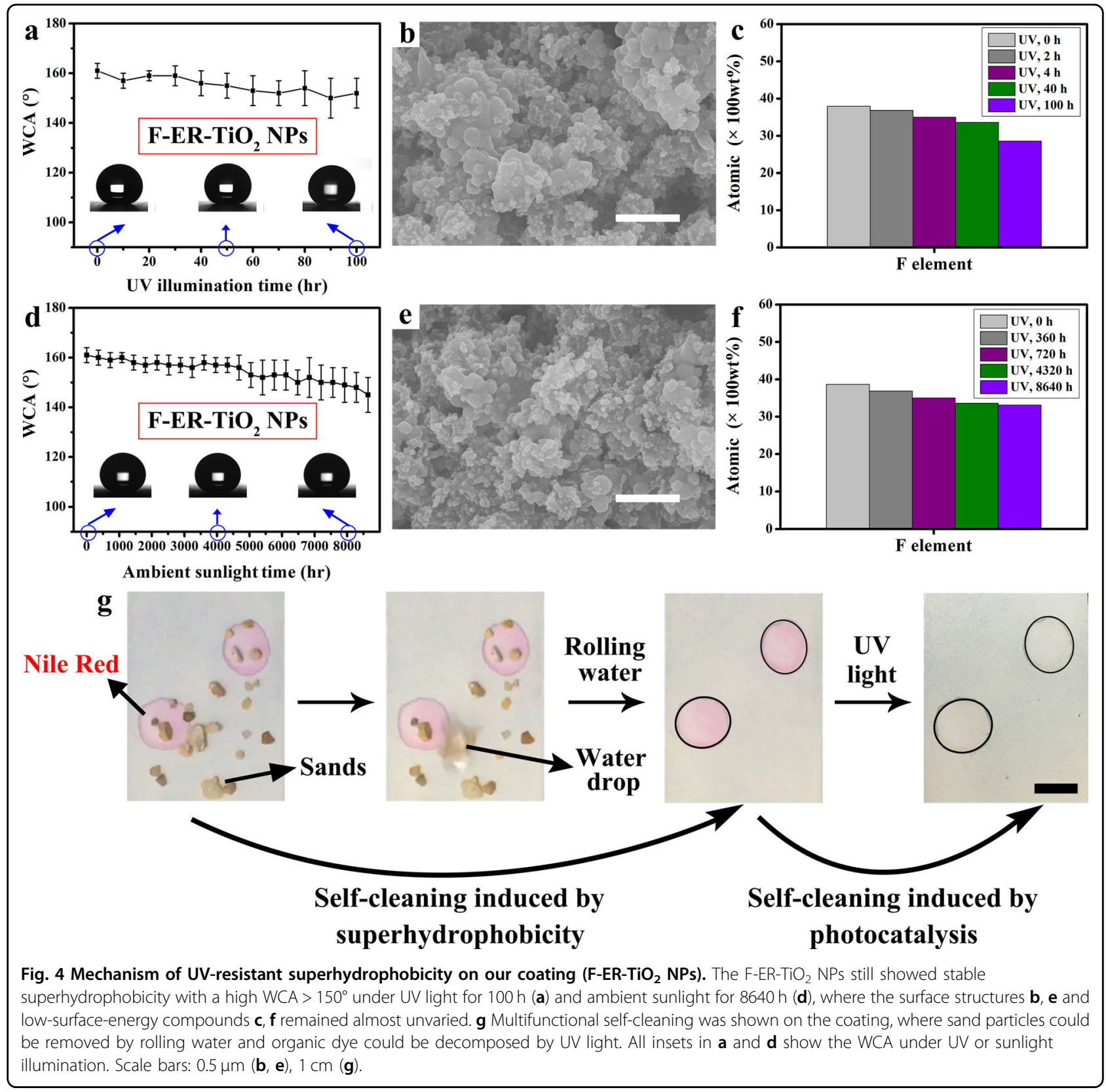

did not perform adequately. Fortunately, the coexistence of superhydrophobicity and photocatalytic properties induced by long-term UV resistance is useful for sustaining a clean surface under such double-polluted conditions. Here, self-cleaning for lotus-inspired dirt removal and organic dye degradation were realized in our coating (Fig. 4g). In Nile red-contaminated areas, super water repellency was also found (Supplementary Fig. S47), where sand with sizes ranging from $0.5 \mathrm{~mm}$ to $2 \mathrm{~mm}$ was freely removed by rolling water droplets (Supplementary Movie S9). Then, organic contaminants were degraded under UV irradiation. Therefore, IOS-PA-coated samples can always remain clean on account of these two kinds of self-cleaning mechanisms.

On rainy days, grass houses are easily wetted, causing considerable trouble for residents in undeveloped regions. Here, we used IOS-PA to coat a simulated grass house (Supplementary Fig. S48). To assess the nonwetting performance, clean and dry papers were placed in grass houses without and with coated IOS-PA (Supplementary Fig. S49). When MB water was poured on the uncoated grass house (Fig. 5a, Supplementary Movie S10), the 


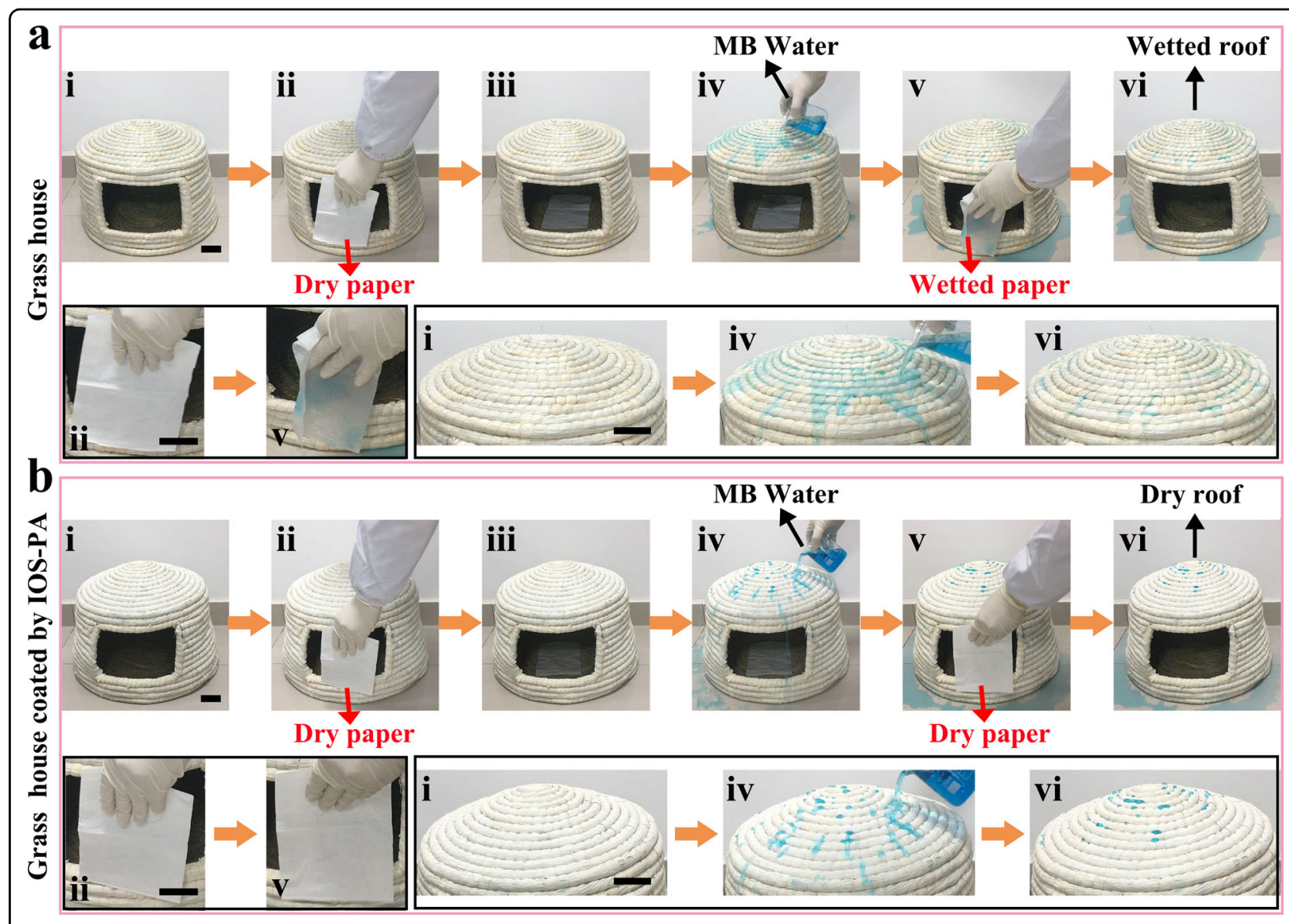

Fig. 5 MB water was poured on the roofs of uncoated and coated grass houses. Water permeated into the roof of the uncoated house and wetted a sheet of dry paper, also leaving a wet and contaminated roof (a). In the coated house, both the paper and roof were dry and clean (b) due to our super-water-repellent paint. All scale bars are $5 \mathrm{~cm}$.

original dry paper became wetted, resulting in a wet and MB-contaminated roof. In contrast, because of the strong water repellency of IOS-PA (Fig. 5b, Supplementary Movie S11), the paper always remained in the dry state, and a waterproof roof was observed.

The costs of the components (ER, curing agent, $\mathrm{TiO}_{2}$ $(21 \mathrm{~nm}), \mathrm{TiO}_{2}(200 \mathrm{~nm})$, PFOS, and acetone) of IOS-PA are shown in Supplementary Table S3. Eighty milliliters of IOS-PA is sufficient to coat a substrate with a surface area of $1 \mathrm{~m}^{2}$, and the cost per unit area for our paint is approximately $2.70 \$ / \mathrm{m}^{2}$, or $17.46 ¥ / \mathrm{m}^{2}$. In addition, the cost of a grass house is approximately $\$ 20$. For a house roof with an area of $100 \mathrm{~m}^{2}$, the total cost to accomplish waterproofing is approximately $\$ 290(270+20)$. As reported by the World Bank, the most poverty-stricken country possesses a per capita GDP of approximately $\$ 303$. Therefore, our reported IOS-PA is affordable for residents in poverty-stricken regions. Furthermore, with its mechanochemical robustness, self-healing properties, high- and low-temperature resistance, anti-icing properties, photocatalytic properties, ultralong UV resistance, and self-cleaning properties, the application of IOS-PA to grass houses exhibits promising and wide potential for real-world applications.

\section{Conclusion}

Multifunctional IOS-PA was fabricated by incorporating dual-sized $\mathrm{TiO}_{2}$ NPs, ER, and PFOS together, showing superior mechanical robustness and chemical stability. Moreover, self-healing properties, high- and lowtemperature $\left(150^{\circ} \mathrm{C}\right.$ and $\left.-196^{\circ} \mathrm{C}\right)$ resistance, and antiicing properties were also observed for our IOS-PA. Photodegradation of organic matter (Nile red, MB and $\mathrm{MO}$ ) was observed on IOS-PA. Moreover, the conflicting properties of photocatalysis and superhydrophobicity were achieved in our paint with long-term functionality, resulting in ultralong UV resistance under UV irradiation $\left(365 \mathrm{~nm}, 5.0 \pm 0.6 \mathrm{~mW} / \mathrm{cm}^{2}\right)$ for $100 \mathrm{~h}$ and ambient sunlight exposure for $8640 \mathrm{~h}$ (1 year). Moreover, IOS-PA showed self-cleaning properties with both dirt removal and organic decomposition. Based on these advantages, grass houses coated with IOS-PA also manifested 
favorable waterproof performance in real life. We believe the reported IOS-PA has the potential for practical production in the near future, as well as daily use for waterproofing items such as roofs, clothes, umbrellas, and shoes.

\section{Acknowledgements}

This work is supported by the National Basic Research Program of China (973 Program, 2015CB932600), the National Key R\&D Program of China (2017YFA0208000, 2016YFF0100800), and the National Natural Science Foundation of China (21525523, 21722507, 21574048, 21874121, 51803194). This research was supported by the Zhejiang Provincial Natural Science Foundation of China under Grant No. LY20B050002. The project was supported by the Fundamental Research Funds for National Universities, China University of Geosciences (Wuhan). This work was supported by the CAS Key Laboratory of Bio-inspired Materials and Interfacial Science, Technical Institute of Physics and Chemistry, Chinese Academy of Sciences. The authors also thank Hui Guo and Yutang Zhu.

\section{Author details}

${ }^{1}$ Engineering Research Center of Nano-Geomaterials of Ministry of Education, Faculty of Materials Science and Chemistry, China University of Geosciences, Wuhan 430074, P. R. China. ${ }^{2}$ Zhejiang Institute, China University of Geosciences, Hangzhou 311305, P. R. China. ${ }^{3}$ Key Laboratory of Catalysis and Energy Materials Chemistry of Education, Hubei Key Laboratory of Catalysis and Materials Science, South-Central University for Nationalities, Wuhan 430074, P. R. China

\section{Author contributions}

H.Z., Y.H., and F.X. conceived and designed the overall experiments. H.Z. and S Z. fabricated the IOS-PA. H.Z. and Y.H. conducted the durability tests. H.Z. and S.J. assessed the XPS data, EPR data, and photocatalysis performance. H.Z., Y.H., X.L., and F.X. wrote and revised the paper. All authors reviewed the manuscript.

\section{Conflict of interest}

The authors declare no competing interests.

\section{Publisher's note}

Springer Nature remains neutral with regard to jurisdictional claims in published maps and institutional affiliations.

Supplementary information The online version contains supplementary material available at https://doi.org/10.1038/s41427-021-00315-x.

\section{Received: 9 November 2020 Revised: 8 March 2021 Accepted: 21 April} 2021.

Published online: 4 June 2021

\section{References}

1. Monitoring Global Poverty (The World Bank, 2017).

2. Meemken, E., Sellare, J., Kouame, C. N. \& Qaim, M. Effects of fairtrade on the livelihoods of poor rural workers. Nat. Sustain. 2, 635-642 (2019).

3. Suzuki, S. \& Manzello, S. L. Initial study on thatched roofing assembly ignition vulnerabilities to firebrand showers. Fire Saf. J. 103, 34-37 (2019).

4. Gao, Z. F. et al. Naked-eye point-of-care testing platform based on a pHresponsive superwetting surface: toward the non-invasive detection of glucose. NPG Asia Mater. 10, 177-189 (2018).

5. Deng, X., Mammen, L., Butt, H. J. \& Vollmer, D. Candle soot as a template for a transparent robust superamphiphobic coating. Science 335, 67-70 (2012).

6. Snapp, P. et al. Interaction of $2 \mathrm{D}$ materials with liquids: wettability, electrochemical properties, friction, and emerging directions. NPG Asia Mater. 12, 22 (2020).

7. Tian, X. L., Verho, T. \& Ras, R. H. A. Moving superhydrophobic surfaces toward real-world applications. Science 352, 142-143 (2016).
8. Zhang, T. Z. et al. Bio-inspired superhydrophilic coatings with high antiadhesion against mineral scales. NPG Asia Mater. 10, e471 (2018).

9. Zhu, Y., Wang, D., Jiang, L. \& Jin, J. Recent progress in developing advanced membranes for emulsified oil/water separation. NPG Asia Mater. 6, e101 (2014).

10. Wang, D. H. et al. Design of robust superhydrophobic surfaces. Nature $\mathbf{5 8 2}$ 55-59 (2020).

11. Pan, S. J. et al. Coatings super-repellent to ultralow surface tension liquids. Nat. Mater. 17, 1040-1047 (2018).

12. Tian, X. C., Shaw, S., Lind, K. R. \& Cademartiri, L. Thermal processing of silicones for green, scalable, and healable superhydrophobic coatings. Adv. Mater. 28, 3677-3682 (2016)

13. Pan, G. M., Xiao, X. Y. \& Ye, Z. H. Fabrication of stable superhydrophobic coating on fabric with mechanical durability, UV resistance and high oil-water separation efficiency. Surf. Coat. Technol. 360, 318-328 (2019).

14. $L u$, Y. et al. Robust self-cleaning surfaces that function when exposed to either air or oil. Science 347, 1132-1135 (2015).

15. Zhi, D. F., Lu, Y., Sathasivam, S., Parkin, I. P. \& Zhang, X. Large-scale fabrication of translucent and repairable superhydrophobic spray coatings with remarkable mechanical, chemical durability and UV resistance. J. Mater. Chem. A $\mathbf{5}$ 10622-10631 (2017).

16. Peng, C. Y., Chen, Z. Y. \& Tiwari, M. K. All-organic superhydrophobic coatings with mechanochemical robustness and liquid impalement resistance. Nat. Mater. 17, 355-360 (2018).

17. Park, J. Y. How titanium dioxide cleans itself. Science $\mathbf{3 6 1}, 753$ (2018).

18. Li, L. H., Bai, Y. Y., Li, L. L., Wang, S. Q. \& Zhang, T. A superhydrophobic smart coating for flexible and wearable sensing electronics. Adv. Mater. 29, 1702517 (2017).

19. Kamegawa, T., Shimizu, Y. \& Yamashita, H. Superhydrophobic surfaces with photocatalytic self-cleaning properties by nanocomposite coating of $\mathrm{TiO}_{2}$ and polytetrafluoroethylene. Adv. Mater. 24, 3697-3700 (2012).

20. Crick, C. R., Bear, J. C., Kafizas, A. \& Parkin, I. P. Superhydrophobic photocatalytic surfaces through direct incorporation of titania nanoparticles into a polymer matrix by aerosol assisted chemical vapor deposition. Adv. Mater. 24, 3505-3508 (2012)

21. Wooh, S., Encinas, N., Vollmer, D. \& Butt, H. J. Stable hydrophobic metal-oxide photocatalysts via grafting polydimethylsiloxane brush. Adv. Mater. 29, 1604637 (2017).

22. Yi, B. et al. Dynamic siloxane materials: from molecular engineering to emerging applications. Chem. Eng. J. 405, 127023 (2021).

23. Liu, M. J. et al. Supramolecular silicone coating capable of strong substrate bonding, readily damage healing, and easy oil sliding. Sci. Adv. 5, eaaw5643 (2019).

24. Li, Y., Chen, S., Wu, M. \& Sun, J. Q. All spraying processes for the fabrication of robust, self-healing, superhydrophobic coatings. Adv. Mater. 26, 3344-3348 (2014).

25. Kreder, M., Alvarenga, J., Kim, P. \& Aizenberg, J. Design of anti-icing surfaces: smooth, textured or slippery? Nat. Rev. Mater. 1, 15003 (2016).

26. He, Z. Y. et al. Bioinspired multifunctional anti-icing hydroge. Matter 2, 1-12 (2019).

27. Jung, S., Tiwari, M. K., Doan, N. V. \& Poulikakos, D. Mechanism of supercooled droplet freezing on surfaces. Nat. Commun. 3, 615 (2012).

28. Rao, K. P., Hiquchi, M., Suryachandram, J. \& Kitagawa, S. Temperature-atable compelled composite auperhydrophobic porous coordination polymers achieved via an unattainable de novo synthetic method. J. Am. Chem. Soc. 140, 13786-13792 (2018).

29. Chen, K. L., Zhou, S. X., Yang, S. \& Wu, L. M. Fabrication of all-wate-based selfrepairing superhydrophobic coatings based on UV-responsive microcapsules. Adv. Funct. Mater. 25, 1035-1041 (2015).

30. Deng, X. et al. Transparent, thermally stable and mechanically robust superhydrophobic surfaces made from porous silica capsules. Adv. Mater. 23 2962-2965 (2011).

31. Meng, A. Y., Zhang, L. Y., Cheng, B. \& Yu, J. G. Dual cocatalysts in $\mathrm{TiO}_{2}$ photocatalysis. Adv. Mater. 31, 1807660 (2019).

32. Schneider, J. et al. Understanding $\mathrm{TiO}_{2}$ photocatalysis: mechanisms and materials. Chem. Rev. 114, 9919-9986 (2014)

33. Bhushan, B. \& Jung, Y. C. Natural and biomimetic artificial surfaces for superhydrophobicity, self-cleaning, low adhesion, and drag reduction. Prog. Mater. Sci. 56, 1-108 (2011).

34. Xia, F. \& Jiang, L. Bio-inspired, smart, multiscale interfacial materials. Adv. Matter 20, 2842-2858 (2008). 
35. Ganesh, V. A., Raut, H. K. Nair, A. S. \& Ramakrishna, S. A review on self-cleaning coatings. J. Mater. Chem. 21, 16304-16322 (2011).

36. Richard, R. D., Clanet, C. \& Quéré, D. Contact time of a bouncing drop. Nature 417, 811 (2002)

37. Bird, J. C., Dhiman, R., Kwon, H. M. \& Varanasi, K. K. Reducing the contact time of a bouncing drop. Nature 503, 385-388 (2013).

38. Liu, Y. H. et al. Pancake bouncing on superhydrophobic surfaces. Nat. Phys. 10 515-519 (2014)
39. $\mathrm{Xu}, \mathrm{Z}$. et al. Recyclable thermoset hyperbranched polymers containing reversible hexahydro-s-triazine. Nat. Sustain. 3, 29-34 (2019).

40. Elzaabalawy, A., Verberne, P. \& Meguid, S. A. Multifunctional silica-silicone nanocomposite with regenerative superhydrophobic capabilities. ACS Appl. Mater. Interfaces 11, 42827-42837 (2019).

41. Yang, H. L. et al. Lotus leaf inspired robust superhydrophobic coating from strawberry-like Janus particles. NPG Asia Mater. 7, e176 (2015) 